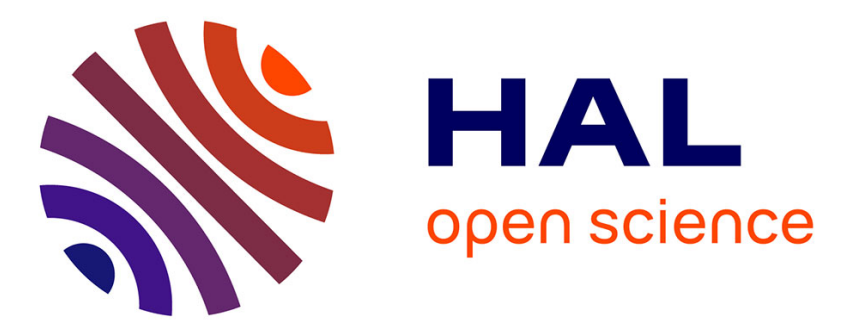

\title{
A Methodology for Efficient Space-Time Adapter Design Space Exploration: A Case Study of an Ultra Wide Band Interleaver
}

\author{
Cyrille Chavet, Philippe Coussy, Pascal Urard, Eric Martin
}

\section{To cite this version:}

Cyrille Chavet, Philippe Coussy, Pascal Urard, Eric Martin. A Methodology for Efficient Space-Time Adapter Design Space Exploration: A Case Study of an Ultra Wide Band Interleaver. The IEEE International Symposium on Circuits and Systems (ISCAS), May 2007, New Orleans, United States. pp.2946-2949, 10.1109/ISCAS.2007.377867 . hal-00153994

\section{HAL Id: hal-00153994 https://hal.science/hal-00153994}

Submitted on 12 Jun 2007

HAL is a multi-disciplinary open access archive for the deposit and dissemination of scientific research documents, whether they are published or not. The documents may come from teaching and research institutions in France or abroad, or from public or private research centers.
L'archive ouverte pluridisciplinaire HAL, est destinée au dépôt et à la diffusion de documents scientifiques de niveau recherche, publiés ou non, émanant des établissements d'enseignement et de recherche français ou étrangers, des laboratoires publics ou privés. 


\title{
A Methodology for Efficient Space-Time Adapter Design Space Exploration: A Case Study of an Ultra Wide Band Interleaver
}

\author{
CHAVET Cyrille ${ }^{1}$, COUSSY Philippe ${ }^{2}$, URARD Pascal $^{1}$, MARTIN Eric $^{2}$ \\ ${ }^{1}$ STMicroelectronics, Crolles, FRANCE. \{firstname.lastname@st.com\} \\ ${ }^{2}$ LESTER Lab, UBS University, CNRS FRE 2734. \{firstname.lastname@univ-ubs.fr\}
}

\begin{abstract}
This paper presents a solution to efficiently explore the design space of communication adapters. In most digital signal processing (DSP) applications, the overall architecture of the system is significantly affected by communication architecture, so the designers need specifically optimized adapters. By explicitly modeling these communications within an effective graph-theoretic model and analysis framework, we automatically generate an optimized architecture, named SpaceTime AdapteR (STAR). Our design flow inputs a $\mathrm{C}$ description of Input/Output data scheduling, and user requirements (throughput, latency, parallelism...), and formalizes communication constraints through a Resource Constraints Graph (RCG). The RCG properties enable an efficient architecture space exploration in order to synthesize a STAR component. The proposed approach has been tested to design an industrial data mixing block example: an Ultra-Wideband interleaver.
\end{abstract}

\section{INTRODUCTION}

The ever growing complexity of applications and the shrinking time-to-market lead the designers to reuse heterogeneous IP cores in Systems-On-a-Chip (SoC) which integration generates communication problems. System integrators can use standard interfaces such as Virtual Component Interface proposed by VSIA [12] and Open Core Protocol proposed by the OCP International Partnership [13]. However, in addition to the protocol aspects, SoC designers also have to synchronize components and to buffer data in order to ensure system behavior and to meet timing constraints. In [10] authors propose to automatically generate simulation wrappers for MPSoC architectures. Based on communication templates, [7] presents a generic interface unit architecture for communication synthesis in a platform-based design approach. In [1] a multiplexer/demultiplexer and FIFO-based interface architecture is used. In [6], the authors propose a systematic way of interfacing dataflow hardware accelerators (IP core) for their integration in a system on chip. Their interface architecture is based on FIFO (queue) storage elements and a Direct Memory Access module (DMA). They assume that the IP are data synchronized (i.e. at each clock cycle a data is presented and read). However, these previous approaches assumed that the sequence of produced data is the same as the sequence of consumed data (no re-ordering). Moreover, FIFO sizes are computed by a "set and simulate" approach.

Concerning Digital Signal Processing (DSP) applications, an MPSoC architecture may not be an adapted solution, and optimized hardware accelerator -composed of a set of computing blocks communicating through point-to-point links- are still needed in the SoC context. Obviously, interfacing DSP's blocks greatly impacts the quality of the system (throughput, area, power consumption...), that's why efficient communication adapter design is still one of the most important points in complex system design. In fact, using Input/Output (I/O) wrappers can introduce unnecessary memorizing elements. Such wrappers may be needed in order to solve data reordering problems that can arise from the IP core integration. In [8] the authors aim at determining at compile time whether a FIFO is sufficient for every producer/consumer pair of a Kahn Process Network. When the sequence of produced data is different from the sequence of consumed data, extra storage and control on the consumer side is proposed [11]. This extra module includes a CAM (Content Addressable Memory) where data are addressed using a hash table. This solution enables the implementation of nondeterministic communications, but it does not allow minimizing of the adapter overhead since overlapping of input and output data is not possible. In [2], a formal technique for hardware interface design is proposed. A generic interface model targeted by the communication synthesis is used. The low-level timing constraints can include strict timing specifications or data transfer schedule. The interface synthesis is carried out by an allocation procedure of data storage components (FIFO, LIFO and register). However, the size of storage elements is not computed or even taken into account during the design process. The proposed methodology is based on NP-complete maximum clique algorithm. In [9] the authors develop a system-level IP reuse methodology where designs are described in three layers. Data transfer and data storage optimizations are done by reorganizing loop indexing and loop nesting. Unfortunately, the authors do not present the technique they use to produce the RTL component architecture from the algorithm specification. In [4], the authors develop a set of techniques dedicated to the design of DSP algorithm. High-level synthesis of the processing unit is carried out under I/O timing and architectural constraints. The approach leads to an optimized data-path synthesis but still requires the communication unit design.

Commercial High Level Synthesis (HLS) tools deal with communication protocol synthesis, but they have a limited communication constraint analysis. So when designers use such tools and want to change communication constraints (throughput, latency, parallelism...), they often have to restart the designing process from the beginning. Some of these tools have no efficient formal model dedicated to communication constraints analysis and to their consequences on the resulting architecture.

In this paper, we present an automatically generated optimized Space-Time AdapteR (STAR). Our design flow inputs a C description of $\mathrm{I} / \mathrm{O}$ data scheduling, and user requirements (throughput, latency...), and formalizes communication constraints through a formal Resource Constraints Graph (RCG). The RCG properties enable an efficient architecture space exploration in order to synthesize a STAR component. The paper is organized as follows: the second section is dedicated to the problem formulation. In the third section we present our design flow, while the associated formal models and methodology are detailed in section four. Finally, the last section presents experimental results.

\section{PROBLEM FORMULATION}

Let us consider a simple architecture example composed of two components exchanging a set of data $S=\{a, b, c, d, e, f\} . S$ is produced by a block \#1 and is consumed by a block \#2 through a single point-to-point link.

The write access sequence into the communication link is $S_{w}=$ $(a, c, b, e, f, d)$ i.e. $t^{w}{ }_{a}<t^{w}{ }_{c}<t^{w}{ }_{b}<t^{w}{ }_{e}<t^{w}<t^{w}{ }_{d}$, while the read access sequence from the link is different $S_{r}=(c, a, e, b, d, f)$ i.e. $t_{t_{c}}^{r}<t^{r}<t^{r}<t^{r}{ }_{b}<t^{r}<t_{f}^{r}$ (see Figure 1). This difference between the two I/O sequences can either come from the integration of two IP cores that were not specifically designed to work together, either can be explicitly described (e.g. in interleavers [5][14]). As those blocks do not produce and consume data in the same order nor with the same 
throughput (nor sometime the same parallelism), they can not be directly plugged together. The designer needs to introduce a spacetime adapter between them to ensure correct functional results. A classical solution consists in using a memory to buffer all concerned data: this is what we call coarse grain approach. But in fact, this over sized buffer may be reduced thanks to a finer grain communication constraints analysis [4]. The proposed adapter can be designed either by using a set of registers or specific memory elements, such as FIFO (queue) or LIFO (stack). The problem the designer faces consists in finding the best architecture for this adapter: he has to find the best storage element binding.

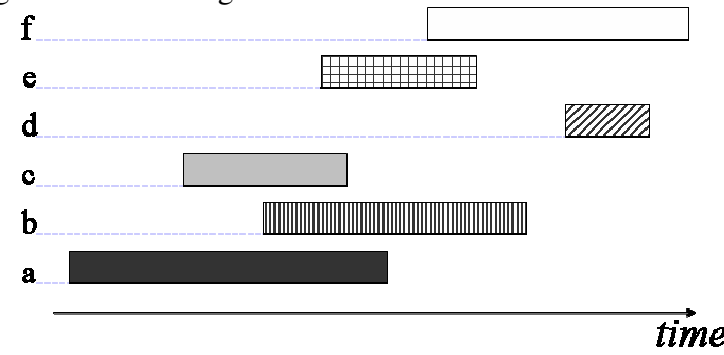

Figure 1: Data lifetime.

For example, the lifetimes of data $a$ and $b$ respect a First-In FirstOut semantic, so they can be assigned to the same hardware FIFO. This timing relation is also true for the data $c$ and $b$. However, data $a$ and $c$ respect a Last-In First-Out semantic, so a single hardware FIFO cannot be used to store the data $a, b$ and $c$. A methodology is thus needed to bind data $a, b$ and $c$ to different storage elements, in order to generate an optimized architecture.

\section{PROPOSED APPROACH}

The architecture of a STAR component is composed of a data path and the associated control state machine FSM (see Figure 2). The data path can be composed of FIFO, LIFO or register. Spatial adaptation (a data read on one input port can be send to any/several output ports) is performed by an interconnection logic dealing with data dispatching from input port to storage elements, and from storage elements to output ports.

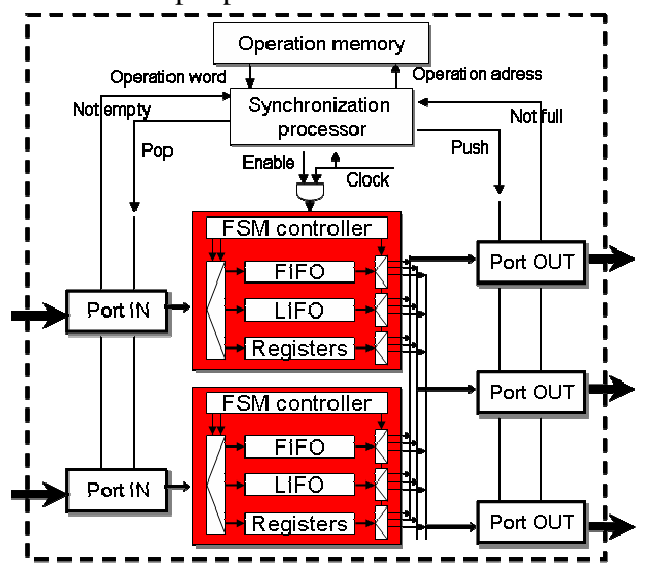

Figure 2: Typical STAR architecture.

The timing adaptation (data-rates, different input/output data scheduling) is realized by the storage elements. STAR can have a GALS (Globally Asynchronous Locally Synchronous) / LIS (Latency Insensitive System) interface as described in [3].

The design flow is presented in Figure 3 and is currently based on three tools: StarTor for the STAR design constraint specification, StarGene for the STAR component synthesis and StarBench for the STAR functional validation. The methodology generates a register transfer level (RTL) architecture starting from a functional model and a set of user requirements (timing and communication-architecture constraints). The architecture synthesis is performed by using a library of pre-designed and characterized storage elements (FIFO, LIFO and Registers).

StarTor inputs a C level algorithmic description which specifies the interleaving scheme, and a file containing user requirements (latency, throughput, communication interface, I/O parallelism...). StarTor first extracts $\mathrm{I} / \mathrm{O}$ data communication order by generating a trace from the execution of the $\mathrm{C}$ functional description. Next, based on designer's requirements, it generates a constraints file. This file contains the number and type of ports, type and amount of data, relationships between data and ports (i.e. mapping) and finally read and write access dates for all data. Then, in order to generate a STAR component, our design tool STARGene is based on a four-step flow: (1) Resource Compatibility Graph construction, (2) Storage resource binding, (3) Architecture optimization and (4) VHDL RTL generation (see Figure 3). During the first step of the STAR component Generation, a Resource Constraints Graph RCG is generated from the communication constraints. The analysis of this formal model allows both data binding to storage elements (queue, stack or register), and the sizing of each storage element. This first architecture is next optimized by merging storage elements that have non-overlapping usage timing frames. Finally, an RTL level design is generated. The last tool, StarBench, generates a test bench based on constraints in order to validate the design by comparing simulation results.

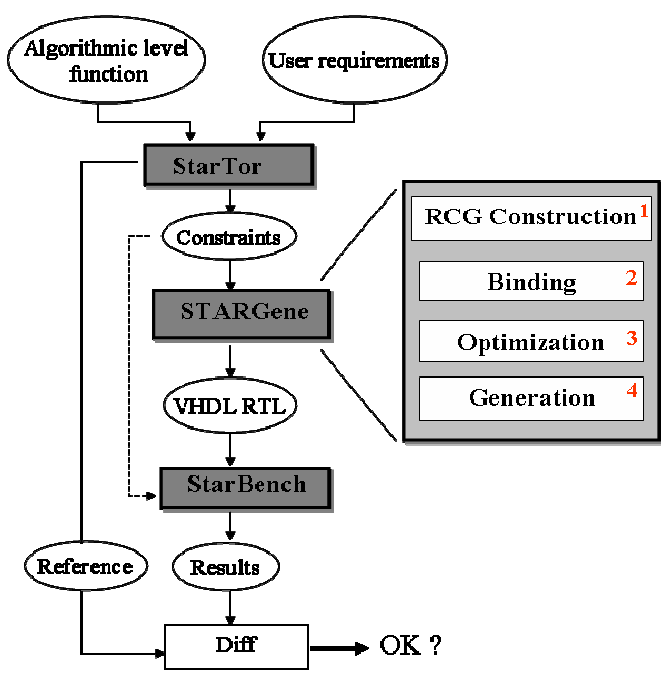

Figure 3: STAR design flow and associated tools.

Typically, a STAR could have to deal with different execution modes (configuration), switching from one to another at run-time. In this paper, we present a formal methodology to synthesize a STAR architecture for a given configuration. The generalization of the methodology generating multi-mode architecture (graph merging, multi data path synthesis, multi FSM generation...) will be presented in a future publication.

\section{STAR DESIGN FLOW}

\section{A. Resource Compatibility Graph Construction}

The first step consists in generating a Resource Compatibility Graph, from the design constraints file. This RCG specifies through formal modeling the timing relationship between data that have to be handled by the STAR architecture. The vertex set $V=\left\{v_{0}, \ldots, v_{n}\right\}$ represents data, the edge set $E=\left\{\left(v_{i}, v_{j}\right)\right\}$ represents the compatibility between the vertices. A tag $t_{i j} \in \mathrm{T}$ is associated with each edge $\left(v_{i}, v_{j}\right)$. This tag represents the compatibility type between the two data ( $i$ and j), $T=\{$ Register $\boldsymbol{R}$, FIFO F, LIFO L $\}$, e.g. Figure 4.

In order to assign compatibility tags to edges, we need to identify the timing relationship that exists between two data. For this purpose 
we defined a set of rules based on functional properties of each storage element (FIFO, LIFO, Register).

The lifetime of data $a$ in a STAR is defined by $\Gamma(a)=\left[\tau_{\min }(a)\right.$, $\tau_{\max }(a)$ ] where $\tau_{\min (a)}$ and $\tau_{\max (a)}$ are respectively the date of the write access of $a$ into the component and the last date of the read access to $a$. $\tau_{\text {first }_{a}}$ is the first read access to $a, \tau_{R i_{a}}$ is the $i$-th read access to $a$ with first $\leq i \leq \max$.

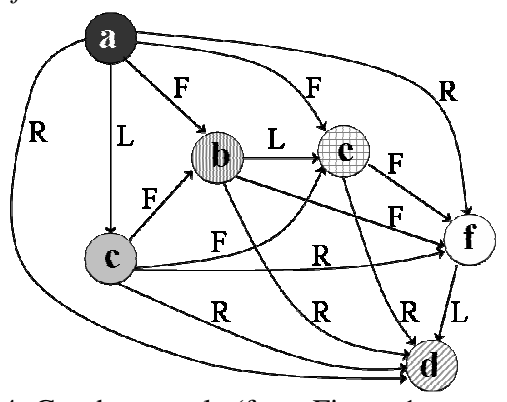

Figure 4: Graph example (from Figure 1 constraints).

Rule 1: Register compatibility

If $\left(\tau_{\text {min }_{b}} \geq \tau_{\text {max }_{a}}\right)$ then we create a "Register" tagged edge.

Rule 2: FIFO compatibility

If $\left[\left(\tau_{\text {min }_{b}}>\tau_{\text {min }_{a}}\right)\right.$ and $\left(\tau_{\text {fist }_{b}}>\tau_{\text {max }_{a}}\right)$ and $\left.\left(\tau_{\text {min }_{b}}<\tau_{\text {max }_{a}}\right)\right]$ then we create a "FIFO" tagged edge.

Rule 3: LIFO compatibility

If $\left[\left[\left(\tau_{\text {min }_{b}}>\tau_{\text {min }_{a}}\right)\right.\right.$ and $\left.\left(\tau_{\text {first }_{a}}>\tau_{\text {max }_{b}}\right)\right]$ or $\left.\left[\left(\tau_{R i_{a}}<\tau_{\text {min }_{b}}<\tau_{\text {max }_{b}}<\tau_{R i+l_{a}}\right)\right]\right]$ then we create a "LIFO" tagged edge

Rule 4: Otherwise, No edge - No compatibility

An analysis of I/O timing relations, we generate a RCG. The graph construction supposes edge creation between data, respecting a chronological order $\left(\tau_{\min }\right)$. If $n$ is the number of data to be handled, the graph may contain: $n(n-1) / 2$ edges, $O\left(n^{2}\right)$.

\section{B. Storage element binding}

The second step consists in binding storage elements to data by using the timing relations modeled by the RCG.

Resource identification: The second step consists in binding storage elements to data by using the timing relations modeled by the RCG. The aim is to identify and to bind as many FIFO or LIFO structures as possible on the RCG.

In [2], by searching and isolating compatibility cliques in an undirected graph, the authors identify the different storage structures (FIFO or LIFO). This approach has four main drawbacks: (1) identifying a maximum clique in an undirected graph is a NPcomplete problem (resource identification step), (2) when such a clique is found, analysis have to be performed to define the clique type (FIFO or LIFO) and to check if the I/O constraints are respected (resource identification step), (3) the proposed flow does not allow sizing of identified storage elements (resource sizing step) and (4) the authors do not propose any exploration algorithm (resource binding step).

Let $a, b, c$ be three chronologically ordered FIFO compatible data $\left(\tau_{\min _{a}}<\tau_{\min _{b}}<\tau_{\min _{c}}\right)$,

\section{Theorem 1}

If $a$ is FIFO compatible with $b$ and $b$ is FIFO compatible with $c$, then $a$ is transitively FIFO (or Register) compatible with $c$.

Theorem 2

If $a$ is LIFO compatible with $b$ and $b$ is LIFO compatible with $c$, then $a$ is transitively LIFO compatible with $c$.

Resource sizing: The size of a LIFO structure equals the maximum number of data stored by a LIFO compatible data path. So, we have to identify the longest LIFO compatibility path $P_{L}$ and then the number of vertices in $P_{L}$ equals the maximum number of data that can be stored in this LIFO (see Figure 5).

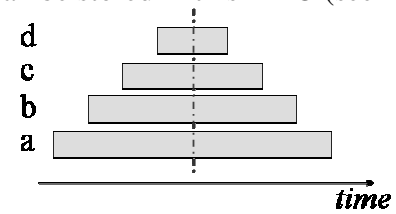

(a) LIFO Gant

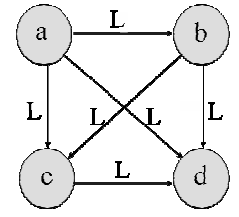

(b) Associated graph
Figure 5: LIFO compatibility cliques.

However, data from a FIFO compatible path are not always FIFO compatible with each other (e.g. Figure 6.a). So the size of a FIFO structure is not always equal to the number of data in the path: the size of the FIFO is the maximum number of data (of the considered path) stored at the same time in the structure. In fact, the aim is to count the maximum number of overlapped data (respecting I/O constraints) in the selected path $P$.

\section{Theorem 3}

Let $\boldsymbol{P}$ be the longest FIFO compatibility path (edges tagged with F), Let $i$ be a vertex of the graph, remaining in $P$,

Let $S_{i}=$ number of incoming FIFO tagged edges, whose origin vertex is in $P$,

Then, $\quad$ Size $=1+\max \left(\left\{S_{i} \mid\right.\right.$ for all vertices $i$ in $\left.\left.P\right\}\right)$.

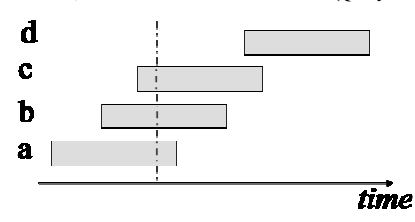

(a) FIFO gantt

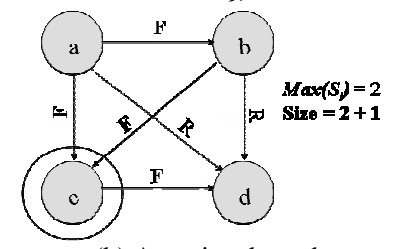

(b) Associated graph
Figure 6: FIFO compatibility cliques.

Resource binding: Our greedy algorithm is based on user plotted metrics (minimal amount of data to use a FIFO or a LIFO, average use factor, FIFO/LIFO usage priority factor...) to bind as many FIFO or LIFO structures as possible on the RCG. A two-steps flow is used: (1) identification of the best structure, (2) merging all the concerned data in a hierarchical node. Each node represents a storage element, as shown on Figure 7.a (e.g. data $a, b$ and $f$ are merged in a 3-stages FIFO). We say hierarchical node because merging a set of data in a given node, supposes adding information that will be useful during the optimization step: the lifetime of this structure (i.e. the time interval during which this structure will be used. e.g. Figure 7.b).

Let $\mathrm{P}=\left\{v_{0}, \ldots, v_{n}\right\}$ be a compatible data path,

- If $\mathrm{P}$ is a FIFO compatible path, the structure lifetime will be $\left[\tau_{\min _{v_{0}}}, \tau_{\max _{v_{n}}}\right]$,

- If $\mathrm{P}$ is a LIFO compatible path, the structure lifetime will be $\left[\tau_{\min _{v_{0}}}, \tau_{\max _{v_{0}}}\right]$.

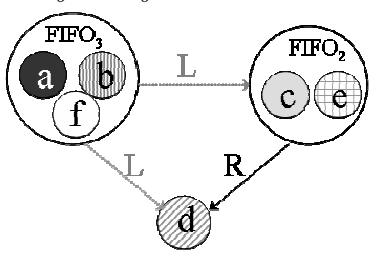

(a) Resulting hierarchical graph

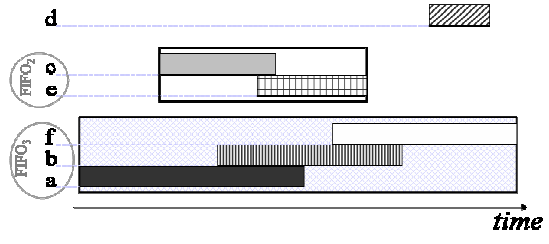

(b) Resulting constraints Figure 7: A possible binding for Figure 4 graph.

The selection of the nodes to be merged in a hierarchical one influences the resulting architecture, since these nodes will not be used to build another structure. When no more FIFO or LIFO structures can be identified on the graph, the next step is architecture optimization.

\section{Architecture Optimization.}


The goal of this task is to maximize storage resource usage, in order to optimize the resulting architecture by minimizing the number of storage elements and the number of structures to be controlled. To tackle this problem, we builded a new hierarchical RCG by using the merged nodes, and their lifetimes, produced during the binding step. In order to avoid any conflict, the exploration algorithm of the optimization step will only search for Register compatibility path, between same type vertices. When two structures of the same type are Register compatible, they can be merged. Let $\mathrm{P}=\left\{v_{0} \ldots v_{n}\right\}$ be a Register compatible data path,

- The lifetime of the resulting hierarchical merged structure will be $\left[\tau_{\min _{v_{0}}}, \tau_{\max _{v_{n}}}\right] \mathrm{U} \ldots \mathrm{U}\left[\tau_{\min _{v_{n}}}, \tau_{\max _{v_{n}}}\right]$.
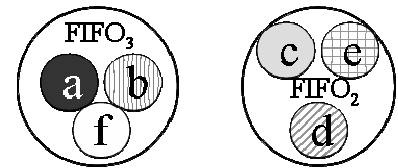

Figure 8: Optimization of Figure 7 graph.

The algorithm is very similar to the one used during binding step. When there is no more merging solution, the resulting graph is used to generate the RTL VHDL architecture. Figure 8 is a possible solution for the constraint set presented in Figure 1. Here, the resulting architecture consist in a 3-stages FIFO that handles 3 data, and a 2-stages FIFO that handles 3 data: one memory place has been saved.

\section{EXPERIMENTS}

In this section we show the results of using our design flow to generate an Ultra Wide Band interleaver [14] example. This component has to be able to switch between different modes (300, 600 or 1200 data length), respecting latency constraints. By nature, interleavers are nearly worst case test-benches for our design flow, since they offer few storage elements to be saved. In a simplistic way, the more the data are interleaved; the better the functional results are for telecommunication applications. However, these datamixing schemes are well-known and very pedagogical mathematical examples and we can explore how metrics (I/O parallelism, enable/disable FIFO/LIFO, average usage factor...) can influence the final architecture.

\begin{tabular}{|c|c|c|c|c|c|c|c|c|}
\hline \multirow[t]{2}{*}{ Mode } & \multicolumn{2}{|c|}{ Reference } & \multicolumn{2}{|c|}{$\begin{array}{c}\text { F/L } \\
(\operatorname{Min} 7 / 95 \%)\end{array}$} & \multicolumn{2}{|c|}{$\begin{array}{c}\text { F/L } \\
(\operatorname{Min} 15 / 90 \%)\end{array}$} & \multicolumn{2}{|c|}{ No F/L } \\
\hline & Saved & Ctrl & Saved & Ctrl & Saved & Ctrl & Saved & Ctrl \\
\hline 300 & $n / a$ & 300 & 56 & 77 & 60 & 240 & 60 & 240 \\
\hline 600 & $n / a$ & 600 & 83 & 101 & 130 & 470 & 130 & 470 \\
\hline 1200 & $n / a$ & 1200 & 96 & 117 & 120 & 609 & 168 & 1032 \\
\hline
\end{tabular}

In Table 1, the number in column saved is the number of register saved, and the number in Ctrl column is the number structure to be managed. Additionnal constraints used during synthesis are F/L minimum length (e.g. 7 or 15) and filling (\%). In the reference architecture there is no memory saving (1200 registers in the worst case, 2400 when pipelined) but the three modes are integrated in a single architecture. Using our flow, we can save registers and decrease latency in any case. The reference design from STMicroelectronics has been generated using a commercial HLS tool.

Moreover the number of structure to be controlled is smaller when we use our model. Drawback of this result is that the reduction of storage elements can increase the complexity of data multiplexing (depending on the interleaving rule). However our approach also enables to enhance the throughput by optimizing the latency to input and next output data. So, depending on the selected mode the throughput of our architecture can vary from 412 to $438 \mathrm{Mb} / \mathrm{s}$ (related to Table 1 designs) compared to $375 \mathrm{Mb} / \mathrm{s}$ as a theoretic throughput from the reference (Table 1).

Currently, we generate the different modes separately, while the reference design integrates the three modes in a single 2400 memory points design. But when we concatenate our three designs (one for each mode) in a single architecture, the total area is about $14 \%$ smaller than the reference design. Future works will enable the generation of optimized multi-modes architectures to further reduce the area.

\section{CONCLUSION}

In this paper, we proposed a design space exploration methodology for Space-Time AdapteR STAR components. This approach relies on the formal modeling of communication constraints based on a Resource Compatibility Graph RCG describing timing relations between data. The binding and optimization steps that assign data to storage elements according to the timing relations have been presented. Experimental results in the telecom domain have demonstrated the interest of this methodology. Formal modeling allows RTL architectures to be synthesized from a single C functional specification and under various I/O timing constraints. We also show that it is easy to explore different solution by applying different constraints during synthesis. This allows enhancements based on refinements. Future works will focus on the formal transformation of the RCG in order to generate multi-configuration and pipelined architectures.

\section{REFERENCES}

[1] F. Abbes, E. Casseau, M. Abid, P. Coussy, J-B. Legof, "IP integration methodology for SoC design", ICM, 2004.

[2] A. Baganne, J-L. Philippe, E. Martin, "A Formal Technique for Hardware Interface design", IEEE Trans. On Circuits And Systems, Vol.45, №5, 1998.

[3] P. Bomel, E. Martin, E. Boutillon, "Synchronization Processor Synthesis for Latency Insensitive Systems", DATE, 2005.

[4] P. Coussy, E. Casseau, P. Bomel, A. Baganne, E. Martin, "A formal method for hardware IP design and integration under I/O and timing constraints", ACM Trans. on Embeded Computing System, 2005.

[5] L. Dinoi, S. Benedetto, "Variable-size interleaver design for parallel turbo decoder architectures", IEEE Transaction on Communication, Vol.53, No11, Nov. 2005.

[6] A. Fraboulet, T. Risset, "Efficient On-chip Communications for Dataflow IPs", ASAP, p.293-303, 2004.

[7] D. Hommais, F. Pétrot, I. Augé, "A Practical Toolbox for System Level Communication Synthesis", In Proceedings of the 9th IEEE International Symposium on Hardware/Software Co-design CODES, 2001.

[8] A. Turjan, et al., "A compile time based approach for solving out-of-order communication in Kahn Process Networks", ASAP, 2002.

[9] F. Vermeulen, F. Catthoor, D. Verkest, H. De Man, "Formalized ThreeLayer System-Level Model and Reuse Methodology for Embedded DataDominated Applications", IEEE Trans. on VLSI Systems, Vol.8, $\mathrm{N}^{\circ} 2,2000$.

[10] S.II Han, A. Baghdadi et al. "An efficient scalable and flexible data transfer architecture for multiprocessor SoC with massive distributed memory", DAC, 2004.

[11]C. Zissulescu-Ianculescu, A. Turjan, B. Kienhuis and E. Deprettere, "Solving Out of Order communication using CAM memory: an implementation", ProRisc, 2002.

[12] Virtual Socket Interface Alliance, http://www.vsi.org.

[13] OCP-IP International Partnership, Open Core Protocol Specification, v2.0, http://www.ocpip.org.

[14] IEEE 802.15.3a, WPAN High Rate Alternative. 